\title{
Learning from Errors While Forecasting Inflation: A Case for Intercept Correction
}

\author{
Muhammad Jahanzeb Malik and Muhammad Nadim Hanif ${ }^{\circledR}$
}

State Bank of Pakistan and State Bank of Pakistan

\begin{abstract}
Structural changes are quite common in macroeconomic time series. Moreover, any underlying macroeconomic relationship cannot be correctly specified unless we know the true model. Structural changes in time series and misspecification in empirical model are observed as shifts in the constant of the underlying relationship between the subject variables of interest. Forecasting from such a model assuming 'no structural break' and 'correct model' is tantamount to ignoring important aspects of underlying economy and mostly results in forecast failure(s). Intercept correction (IC) is a method for accommodating such ignored structural break(s) and omitted variable(s). We use a simple model (for July 1991 to March 2016) to forecast inflation for 25 countries and compare its performance with a) the same model with optimal IC, b) the same model with half IC, and c) a random walk model. Optimal IC approach, though computational intensive, outperforms in forecasting next period inflation compared to one from a) the same model without IC, b) the same model with half intercept correction, and c) random walk model without IC. For the particular class of inflation models under study, over the time period specified, 'quarter IC' works best among the fixed IC rules.
\end{abstract}

Key words: Forecasting, Structural changes, Intercept correction, Misspecification, Inflation models

JEL Classifications: C01, C52, C53

\section{INTRODUCTION}

The forward-looking nature of monetary policy highlights the importance of inflation forecasting. Accordingly, we need to be aware of the main factors behind the forecast failures. Mean shift or intercept change is considered as the biggest enemy of forecasts Clements and Hendry (1999). This study primarily highlights the importance of intercept correction (IC) and describes a framework for its implementation for details; see Clements and Hendry (1999). For illustration, we use a simple model of inflation (as a function of lagged broad money supply growth). Even though this appears to be a naïve model, the intercept correction method protects against various forms of mis-specification errors in complex models and compensates for the effects of missing variables. Thus, it can prove to be a strong competitor to sophisticated models. However, what should be the degree of IC, between zero IC to unit IC, is the research question of this study.

We estimate a simple inflation forecasting model (which is equivalent to 'zero intercept correction' or ZIC) and compare its performance with a random walk model (RWM) as

\footnotetext{
${ }^{\circledR}$ Muhammad Nadim Hanif, (email: muhammadnadeemhanif@ yahoo.com), Tel: +923212436714.

Muhammad Jahanzeb Malik, (email: jazeephd@ gmail.com), Tel: +923215018594.

Authors belong to State Bank of Pakistan. The views expressed in this study are those of the authors and need not necessarily be associated with their employer. Authors are thankful to the Editor for his insightful comments on earlier version of this study which helped improved this a lot.
} 
benchmark. We also compare the performance of ZIC with the same but allowing for some non-zero fraction of IC. The degree of IC could be incorporated in different ways. First, arbitrarily selecting a fraction of previous forecast error (PFE) which is obviously the simple and stable approach to decide a fraction of error to be corrected. Second, an optimal selection of a fraction of PFE which is a sophisticated way to estimate a fraction of error to be corrected but may vary with the sample. We explore both of these ways for IC in this study. For an arbitrary selection of the 'fraction' or 'weight' (w) of the PFE, we choose it to be 0.5 . This we call half intercept correction (HIC) rule. Similarly, one may have a quarter intercept correction (QIC) rule or three-quarters intercept correction rule. For optimal selection of this weight (w), we search for the one which minimizes the root mean squared error (RMSE) of in-sample inflation forecasts. We denote this correction by OIC.

For this study we select 25 countries (including Pakistan). In case of Pakistan we also make such comparisons (forecasting with and without IC) for a larger set of inflation forecasting models which we studied in Hanif and Malik (2015).

For all 25 countries, OIC beats ZIC, HIC as well as RW model (of inflation). In case of Pakistan, OIC based forecasting beats all 9 inflation forecasting models in Hanif and Malik (2015) including highly technical models of inflation with seemingly strong theoretical background. While selecting the optimal degree of intercept correction $(w)$ we observe that a higher intercept correction is needed for the countries where inflation is volatile (and high). For the particular class of inflation forecasting models under investigation, over the period studied in this paper, 'quarter intercept correction (QIC)' works best among the fixed intercept correction rules.

In the next section, we explain the data and the methodology we use. In section 3 we provide empirical evidence in accordance with the methodology outlined. Last section is for concluding remarks.

\section{METHODOLOGY AND DATA}

A variant of the quantity theory of money suggests inflation (deflation) results from expansion (contraction) in money supply in an economy. In order to demonstrate the usefulness of intercept correction see Clements and Hendry (1999), we start with this simple relationship to forecast inflation while considering other competing determinants of inflation including global commodity prices (which we proxy by international prices of crude oil), nominal exchange rate, and real economic activity in the country. We model general level of price change (measured by CPI) as a function of optimal lag(s) of inflation itself and optimal lags of broad money supply growth, international crude oil price change, change in nominal exchange rate, and a proxy for changes in real economic activity. We collect monthly data for all the variables from International Financial Statistics of IMF for 25 countries. The change can be monthly (i.e. month on month, denoted by MoM) or annual (i.e. year on year denoted by YoY). For MoM case, as an example, let us denote $y_{t}=\log \left(C P I_{t} / C P I_{t-1}\right)$ and $x_{t}=\log \left(M 2_{t} / M 2_{t-1}\right)$. Similarly, we can define the other set of variables $z_{t}$, if any, such as $\log \left(E X C R_{t} / E X C R_{t-1}\right)$ for exchange rate, and so on; $z_{t}$ could be different from the true model (as we do not know the true model).

Dividing the available dataset into two halves, we run the simple linear regression [using linest, in Excel] without any constant as in equation (2.1), i.e.,

and thus,

$$
\mathrm{y}_{\mathrm{t}}=\mathrm{b}_{0} \mathrm{y}_{\mathrm{t}-\mathrm{i}}+\mathrm{c}_{0} \mathrm{x}_{\mathrm{t}-\mathrm{j}}+\mathrm{d}_{0} \mathrm{z}_{\mathrm{t}-\mathrm{k}}+\cdots+\epsilon_{\mathrm{t}}
$$




$$
y_{t}=\hat{b}_{0} y_{t-i}+\hat{c}_{0} x_{t-j}+\widehat{d}_{0} z_{t-k}+\cdots+\widehat{\epsilon}_{t}
$$

or,

$$
\mathrm{y}_{\mathrm{t}}-\left\{\hat{\mathrm{b}}_{0} \mathrm{y}_{\mathrm{t}-\mathrm{i}}+\widehat{\mathrm{c}}_{0} \mathrm{x}_{\mathrm{t}-\mathrm{j}}+\widehat{\mathrm{d}}_{0} \mathrm{z}_{\mathrm{t}-\mathrm{k}}+\cdots\right\}=\widehat{\epsilon}_{\mathrm{t}}
$$

where $i, j$, and $k$ can be chosen on the basis of either some lag selection criterion or some prior information or a combination of both approaches. Since our focus here is not lag selection and we want to explore the merits of IC only, we do not go into lag selection related details here. However, even if the lag selection is neither optimal nor based upon any prior information, intercept correction takes care of lag selection related misspecification, in addition to omitted variable(s) bias, at least partially (because the misspecification can all be considered as a fluctuation in the constant intercept, to a first order of approximation).

For this study, we use dataset from July 1991 to March 2016. If we denote the sample size used in this estimation as $T^{*}$ (which is half of the overall sample size T), we can see that the prediction error at $t=T^{*}$ from equation (2) is as below:

$$
\mathrm{y}_{\mathrm{t}}=\hat{\mathrm{b}}_{0} \mathrm{y}_{\mathrm{t}-\mathrm{i}}+\hat{\mathrm{c}}_{0} \mathrm{x}_{\mathrm{t}-\mathrm{j}}+\widehat{\mathrm{d}}_{0} \mathrm{z}_{\mathrm{t}-\mathrm{k}}+\cdots+\widehat{\epsilon}_{\mathrm{t}}
$$

The intercept correction method uses the forecast error from the current period to adjust the forecast in the next period by a fraction, say, $w_{0}$. The adjusted forecast at $t=T^{*}+1$, i.e., $y_{T^{*}+1}$ is

$$
y_{T^{*}+1}^{F}=\hat{b}_{0} y_{T^{*}}+\hat{c}_{0} x_{T^{*}}+\hat{d}_{0} z_{T^{*}}+\cdots+\widehat{w}_{0} \widehat{\epsilon}_{T^{*}}
$$

This has the forecast error: $y_{T^{*}+1}-y_{T^{*}+1}{ }^{F}=\in^{F} T^{*}+1$. When $w_{0}=0$, this is the usual forecast, without intercept correction. If it is anticipated that the next period forecast will contain an error close to quarter, half, or three-quarters of the error made in the previous period then quarter, half, or three-quarters fraction of PFE can be adjustment respectively to account for the 'anticipated' error.

The above process is repeated by adding one observation and so on. We go until $t=T$ i.e. from $T^{*}$ to $T$. Forecast $y^{F} T^{*}+2$ and the corresponding forecast errors are $\in_{T^{*}+2}$ and so on are obtained accordingly.

Using $\in^{F}{ }_{T^{*}+1} \ldots \ldots \ldots \ldots \ldots \ldots \ldots \ldots \in^{F}$, ee calculate root mean square error (RMSE) of forecasts as

$$
\operatorname{RMSE}(y)=\sqrt{\frac{\sum_{t=T^{*}}^{T}\left(\epsilon^{F}\right)^{2}}{T-T^{*}}}
$$

In order to judge if quarter, half, or three-quarters ${ }^{1}$ intercept correction should be adopted as a fractional intercept correction rule, we should see which fractional adjustment results in RMSE within, say, $10 \%$ of the minimum RMSE to forecast inflation rate for a given country.

For optimal intercept correction, we repeat the above procedure for $w_{0}=$ $0,0.01, \ldots \ldots \ldots \ldots \ldots \ldots, 0.99,1.0$ and look for the degree of interception that yields minimum RMSE.

For the case of empirical exercise in this study, Model (2.1) is estimated using data from July 1991 to October 2003 (first half of dataset), we then recursively forecast inflation from November 2003 to March 2016 (second half of dataset). At each step one observation is increased in the estimation sample and PFE is added with some coefficient "wo" in the model

\footnotetext{
${ }^{1}$ Or, any arbitrary value that can be fixed as a rule for fractional intercept correction.
} 
and we estimate model represented in equation (2.3). This process is repeated for all observations till final forecast of March 2016 is obtained. In order to find out the optimal value of $\mathrm{w}_{0}$, RMSE is calculated for $w_{0}=0,0.01$, $, 0.99,1.0$. Optimal value of " $w_{0}$ " is obtained where RMSE of forecasts is minimized and is called OIC fraction.

\section{ESTIMATION AND RESULTS}

Since we are working with growth data (of prices and money), we do not expect our variables of interest to be non-stationary and thus do not test for unit roots. Moreover, the benchmark forecasting model (RW or AR1) works with and without unit roots and thus we need not worry about stationarity. As discussed, we estimate i) a benchmark model, ii) simple inflation forecasting model (without intercept), iii) an inflation forecasting model with adding back half of the forecasting error in underlying ZIC model (ii), and iv) an inflation forecasting model with the addition of an optimal degree of intercept correction back to the underlying ZIC model. For benchmarking purpose we estimate a random walk model of inflation with drift as found to be the best benchmarking model in forecasting inflation in Pakistan by Hanif and Malik (2015).

\begin{tabular}{lccc}
\hline \hline Country & Optimal degree of IC & $\begin{array}{c}\text { RRMSE } \\
\text { (viz a viz RW WD) }\end{array}$ & $\begin{array}{c}\text { RRMSE } \\
\text { (viz a viz HIC) }\end{array}$ \\
\hline Bolivia & 0.52 & 0.50 & 0.50 \\
Colombia & 0.89 & 0.65 & 0.68 \\
Costa Rica & 0.46 & 0.50 & 0.50 \\
Denmark & 0.23 & 0.52 & 0.53 \\
Dominica & 0.00 & 0.39 & 0.44 \\
Fiji & 0.00 & 0.38 & 0.43 \\
Grenada & 0.29 & 0.44 & 0.45 \\
Japan & 0.28 & 0.50 & 0.51 \\
Jordan & 0.21 & 0.44 & 0.46 \\
Malaysia & 0.41 & 0.52 & 0.52 \\
Mexico & 0.79 & 0.61 & 0.63 \\
Pakistan & 0.13 & 0.43 & 0.45 \\
Paraguay & 0.16 & 0.41 & 0.43 \\
Singapore & 0.00 & 0.40 & 0.43 \\
South Africa & 0.38 & 0.52 & 0.53 \\
St Kitts \& Nevis & 0.04 & 0.42 & 0.45 \\
St Lucia & 0.04 & 0.40 & 0.44 \\
St Vincent \& Grenadines & 0.00 & 0.39 & 0.43 \\
Swaziland & 0.19 & 0.37 & 0.39 \\
Switzerland & 0.11 & 0.46 & 0.47 \\
Tonga & 0.07 & 0.42 & 0.45 \\
Trin/Tobago & 0.14 & 0.45 & 0.47 \\
Turkey & 0.59 & 0.54 & 0.54 \\
U.S. & 0.65 & 0.59 & 0.60 \\
Uruguay & 0.49 & 0.52 & 0.52 \\
\hline S.1 Reate RMSE of OIC & & & \\
\hline
\end{tabular}

Table 3.1 Relative RMSE of OIC model with respect to i) random walk with drift and ii) HIC model Note: These results pertain to MoM dataset.

In Table 3.1, we report the relative RMSE of an inflation forecasting model, as in (2.1), with optimal value of $\mathrm{w}_{0}$ for all countries. We study RMSE relative to RMSE of RW model with drift and HIC model. Optimal intercept corrected version performs well in all cases. These results are robust to whether we consider MoM or YoY style of calculating the growth. More importantly, these results are robust to whether or not we use the additional set of potential 
explanatory variables $\left(z_{t}\right)$ of inflation ${ }^{2}$ simply because when we omit some of the relevant explanatory variable(s) their changes reflect in the intercept and intercept correction (partially) compensates for the omitted variable(s).

In all above analysis pertaining to performance of inflation forecasting models with intercept correction, we have quite interesting observations: The optimal degree of intercept correction is different for different countries (Table 3.2a). It suggests exploring which type of countries requires relatively a higher degree of intercept correction. On plotting the optimal degree of intercept correction with the degree of volatility in inflation we observe that both are directly related - higher the volatility in inflation, higher would be the required optimal degree of intercept correction in order to forecast inflation best (Figure B.1). It highlights the importance of using intercept correction in inflation forecasting particularly for high inflation countries because countries with volatile inflation are usually the high inflation countries. For each country the OIC does not vary much over extending the sample period as is clear from the last column of Table 3.2a. It shows very low standard deviations of OICs for different samples barring for pre global commodity prices shock of 2007-08. This is also clear from 'closeness' of country wise OICs plotted in Figure B.6 (Appendix B) for different samples.

\begin{tabular}{|c|c|c|c|c|c|c|c|c|c|}
\hline Country & $\begin{array}{c}1992- \\
2008\end{array}$ & $\begin{array}{l}1992- \\
2012\end{array}$ & $\begin{array}{l}1992- \\
2013\end{array}$ & $\begin{array}{c}1992- \\
2014\end{array}$ & $\begin{array}{l}1992- \\
2015\end{array}$ & $\begin{array}{c}\text { 1992- } \\
2016 \\
\text { (March) }\end{array}$ & $\begin{array}{c}\text { Average } \\
\text { (columns } \\
2 \text { to } 8 \text { ) }\end{array}$ & $\begin{array}{l}\text { Standard } \\
\text { Deviation } \\
\text { (columns } \\
2 \text { to } 8 \text { ) }\end{array}$ & $\begin{array}{c}\text { Standard } \\
\text { Deviation } \\
\text { (columns } \\
3 \text { to 8) }\end{array}$ \\
\hline (1) & $(2)$ & (3) & (4) & $(5)$ & (6) & $(7)$ & $(8)$ & (9) & (9) \\
\hline Bolivia & 0.21 & 0.39 & 0.38 & 0.40 & 0.45 & 0.52 & 0.39 & 0.10 & 0.10 \\
\hline Colombia & 0.71 & 0.83 & 0.81 & 0.87 & 0.87 & 0.89 & 0.83 & 0.07 & 0.07 \\
\hline Costa Rica & 0.23 & 0.42 & 0.40 & 0.41 & 0.44 & 0.46 & 0.39 & 0.08 & 0.08 \\
\hline Denmark & 0.05 & 0.27 & 0.22 & 0.25 & 0.20 & 0.23 & 0.20 & 0.08 & 0.08 \\
\hline Dominica & 0.16 & 0.00 & 0.00 & 0.00 & 0.00 & 0.00 & 0.03 & 0.07 & 0.07 \\
\hline Fiji & 0.16 & 0.00 & 0.00 & 0.00 & 0.00 & 0.00 & 0.03 & 0.07 & 0.07 \\
\hline Grenada & 0.06 & 0.34 & 0.36 & 0.34 & 0.34 & 0.29 & 0.29 & 0.11 & 0.11 \\
\hline Japan & 0.13 & 0.27 & 0.26 & 0.28 & 0.31 & 0.28 & 0.26 & 0.06 & 0.06 \\
\hline Jordan & 0.13 & 0.19 & 0.23 & 0.23 & 0.24 & 0.21 & 0.21 & 0.04 & 0.04 \\
\hline Malaysia & 0.33 & 0.36 & 0.39 & 0.38 & 0.41 & 0.41 & 0.38 & 0.03 & 0.03 \\
\hline Mexico & 0.52 & 0.77 & 0.79 & 0.78 & 0.77 & 0.79 & 0.74 & 0.11 & 0.11 \\
\hline Pakistan & 0.26 & 0.13 & 0.12 & 0.09 & 0.11 & 0.13 & 0.14 & 0.06 & 0.06 \\
\hline Paraguay & 0.29 & 0.21 & 0.20 & 0.20 & 0.18 & 0.16 & 0.21 & 0.04 & 0.04 \\
\hline Singapore & 0.00 & 0.00 & 0.00 & 0.00 & 0.00 & 0.00 & 0.00 & 0.00 & 0 \\
\hline South Africa & 0.42 & 0.38 & 0.31 & 0.31 & 0.33 & 0.38 & 0.36 & 0.05 & 0.05 \\
\hline St Kitts \& Nevis & 0.27 & 0.12 & 0.11 & 0.04 & 0.04 & 0.04 & 0.10 & 0.09 & 0.09 \\
\hline St Lucia & 0.03 & 0.01 & 0.00 & 0.00 & 0.00 & 0.00 & 0.01 & 0.01 & 0.01 \\
\hline St Vincent \& Gr. & 0.00 & 0.00 & 0.03 & 0.00 & 0.00 & 0.00 & 0.01 & 0.01 & 0.01 \\
\hline Swaziland & 0.37 & 0.20 & 0.19 & 0.09 & 0.15 & 0.19 & 0.20 & 0.09 & 0.09 \\
\hline Switzerland & 0.00 & 0.00 & 0.00 & 0.06 & 0.08 & 0.11 & 0.04 & 0.05 & 0.05 \\
\hline Tonga & 0.00 & 0.05 & 0.09 & 0.14 & 0.07 & 0.07 & 0.07 & 0.05 & 0.05 \\
\hline Trin/Tobago & 0.00 & 0.20 & 0.14 & 0.13 & 0.14 & 0.14 & 0.13 & 0.07 & 0.07 \\
\hline Turkey & 0.63 & 0.62 & 0.61 & 0.60 & 0.62 & 0.59 & 0.61 & 0.01 & 0.01 \\
\hline$U . S$. & 0.52 & 0.59 & 0.59 & 0.60 & 0.64 & 0.65 & 0.60 & 0.05 & 0.05 \\
\hline Uruguay & 0.91 & 0.60 & 0.57 & 0.53 & 0.51 & 0.49 & 0.60 & 0.16 & 0.16 \\
\hline
\end{tabular}

Table 3.2a Optimal degree of intercept correction estimated for different time periods Note: These results pertain to MoM dataset.

For the case of Pakistan specifically, we also compare the performance of optimal intercept correcting the inflation-forecasting model of this study with a number of inflation forecasting

\footnotetext{
${ }^{2} z_{t}$ includes global commodity prices, nominal exchange rate, and real economic activity. Results pertaining to case without $z_{t}$ can be obtained from the corresponding author, if needed.
} 
models estimated by Hanif and Malik (2015). In Table 3.3, we report the RMSE of 9 inflation forecasting models of Hanif and Malik (2015) relative to RMSE of OIC model of this study. Thus, study's OIC inflation forecasting model performs better compared to all the models we considered from Hanif and Malik (2015).

\begin{tabular}{|c|c|c|c|c|c|c|c|}
\hline Country & OIC groups & $\begin{array}{l}1992- \\
2008 \\
\end{array}$ & $\begin{array}{l}1992- \\
2012 \\
\end{array}$ & $\begin{array}{l}1992- \\
2013 \\
\end{array}$ & $\begin{array}{l}1992- \\
2014 \\
\end{array}$ & $\begin{array}{l}1992- \\
2015 \\
\end{array}$ & $\begin{array}{c}1992- \\
2016 \text { (March) } \\
\end{array}$ \\
\hline 1 & \multirow{5}{*}{0} & 0 & 0 & 0 & 0 & 0 & 0 \\
\hline 2 & & 0 & 0 & 0 & 0 & 0 & 0 \\
\hline 3 & & 0 & 0 & 0 & 0 & 0 & 0 \\
\hline 4 & & 0 & 0 & 0 & 0 & 0 & 0 \\
\hline 5 & & 0 & 0 & 0 & 0 & 0 & 0 \\
\hline 6 & \multirow{9}{*}{ Above 0 but below 0.25} & 0.03 & 0.01 & 0.03 & 0.04 & 0.04 & 0.04 \\
\hline 7 & & 0.05 & 0.05 & 0.09 & 0.06 & 0.07 & 0.07 \\
\hline 8 & & 0.06 & 0.12 & 0.11 & 0.09 & 0.08 & 0.11 \\
\hline 9 & & 0.13 & 0.13 & 0.12 & 0.09 & 0.11 & 0.13 \\
\hline 10 & & 0.13 & 0.19 & 0.14 & 0.13 & 0.14 & 0.14 \\
\hline 11 & & 0.16 & 0.2 & 0.19 & 0.14 & 0.15 & 0.16 \\
\hline 12 & & 0.16 & 0.2 & 0.2 & 0.2 & 0.18 & 0.19 \\
\hline 13 & & 0.21 & 0.21 & 0.22 & 0.23 & 0.2 & 0.21 \\
\hline 14 & & 0.23 & & 0.23 & 0.25 & 0.24 & 0.23 \\
\hline 14 & \multirow{7}{*}{ Above 0.25 but below 0.50} & & 0.27 & & & & \\
\hline 15 & & 0.26 & 0.27 & 0.26 & 0.28 & 0.31 & 0.28 \\
\hline 16 & & 0.27 & 0.34 & 0.31 & 0.31 & 0.33 & 0.29 \\
\hline 17 & & 0.29 & 0.36 & 0.36 & 0.34 & 0.34 & 0.38 \\
\hline 18 & & 0.33 & 0.38 & 0.38 & 0.38 & 0.41 & 0.41 \\
\hline 19 & & 0.37 & 0.39 & 0.39 & 0.4 & 0.44 & 0.46 \\
\hline 20 & & 0.42 & 0.42 & 0.4 & 0.41 & 0.45 & 0.49 \\
\hline 21 & \multirow{4}{*}{ Above 0.50 but below 0.75} & 0.52 & 0.59 & 0.57 & 0.53 & 0.51 & 0.52 \\
\hline 22 & & 0.52 & 0.6 & 0.59 & 0.6 & 0.62 & 0.59 \\
\hline 23 & & 0.63 & 0.62 & 0.61 & 0.6 & 0.64 & 0.65 \\
\hline 24 & & 0.71 & & & & & \\
\hline 24 & \multirow{2}{*}{ Above 0.75} & & 0.77 & 0.79 & 0.78 & 0.77 & 0.79 \\
\hline 25 & & 0.91 & 0.83 & 0.81 & 0.87 & 0.87 & 0.89 \\
\hline
\end{tabular}

Table 3.2b Distribution of Optimal degree of intercept correction estimated for different time periods Note: These results pertain to MoM dataset.

\begin{tabular}{lc}
\hline \hline Models & Relative RMSE \\
\hline ARIMA & 0.76 \\
MVAR & 0.87 \\
EVAR -1 & 0.20 \\
EVAR -2 & 0.58 \\
CVAR & 0.31 \\
MVAR (Bayesian) & 0.75 \\
EVAR (Bayesian) & 0.73 \\
CVAR (Bayesian) & 0.71 \\
ARDL & 0.96 \\
\hline \hline
\end{tabular}

Table 3.3 Relative Forecast Performance (Relative to Intercept Correction Model)

When we compare the performance of different fixed intercept correction rules (like HIC), we find that QIC works best amongst quarter, half and three-quarters intercept correction.

\section{CONCLUSION}

We use a simple model of inflation consisting of lag(s) of inflation along with lags of broad money supply growth to forecast next period inflation. We evaluate forecasting performance of 
this model in comparison with a random walk model of inflation. Then we see if intercept correction (IC) helps improve the inflation forecasting performance of this model in comparison with the performance of the same model with no IC. An IC can also be applied arbitrarily like half intercept correction (HIC). However, we think a 'root mean squared error (RMSE) minimizing approach' can be adopted to decide for the desired degree of IC rather than no or arbitrary IC. We call such degree of IC as an optimal intercept correction (OIC). 'Is really the idea of OIC worthwhile compared to no or arbitrarily selected degree of IC' is the one of the two question of this study. The other question is which degree of interception correction works best from among the different fixed intercept correction rules like quarter, half, etc.

We carry out an exercise for 25 countries over a period from July 1991 to March 2016 during which we know inflation rate series are characterized by different structural breaks (including the one during 2008). The simple model of inflation works for all countries against random walk model as there is no such country for which relative RMSE is greater than unity (Figure B.2-B.5 of Appendix B) irrespective of fraction of IC- like no, half or optimal.

Three main results are interesting.

No intercept correction is not an option as we can see that zero IC is not optimal for 20 out of 25 countries. In other words, minimum relative RMSE is achieved only for 5 out of 25 countries (Dominica, Fiji, Singapore, and St. Vincent and Grenadines) when no IC is applied. There can be a couple of countries for which minimum relative RMSE occurs for fraction of IC which may be statistically not different from zero (like 0.04 in the cases of St. Kitts and Nevis and St. Lucia). One can interpret this result as if intercept correction does not really help improve the inflation forecast for these 7 countries. However, the proportion of such countries where no intercept correction is required is found to be small. In most of the cases improving inflation forecast needs intercept correction. Likewise, complete or full intercept correction is also not an option as we cannot found even a single country where unit IC is optimal.

OIC works for most of the countries against no or half intercept correction model of inflation forecasting (Figure B.2-B.5 of Appendix B). The value of OIC found stable for respective countries when we estimate it by increasing the sample size for one year over a period of four years (from 1992-2012 to 2008-2013, 2008-2014, 2008-2015 and 2008-2016) as shown in Table 3.2a. The idea of OIC is worthwhile for forecasting purpose that too with or without inclusion of available relevant explanatory variable(s) for the variable of interest (inflation in this study). Once we observe different optimal degree of IC for different countries, we also observe that higher degree of IC is required for better forecasts of inflation rate in economies with relatively volatile inflation rate. OIC based simple inflation forecasting model for Pakistan also performs best compared to all the relatively sophisticated models we estimated in Hanif and Malik (2015) for inflation forecasting purpose.

In case one thinks selecting an OIC is estimation intensive, s/he may use the simplest approach of selecting an arbitrary fraction of IC. From among the various options of fixing the fraction of intercept correction, say one quarter, half or three quarters, different fractions perform differently. HIC does not produce minimum RMSE for 22 out 25 countries. Minimum RMSE is achieved only for 3 out of 25 countries (Bolivia, Costa Rica and Japan) when HIC is applied. Again, there can be a couple of countries for which minimum relative RMSE occurs for fraction of IC which may be statistically not different from half (like 0.52 for the cases of South Africa and Uruguay). Based on our observation in this study - that in most of the cases OIC falls close 
to 0.25 (Table 3.2b) compared to only a few (a couple of) cases falling close to half (threefourths) intercept correction - QIC may be a preferred choice to create a fixed IC rule.

In the end, we would like to lodge a caveat. Our study is based upon one relationship only and there is need to attempt this investigation for some other relationships as well.

\section{REFERENCES}

Castle, J.L., M.P. Clements and D.F. Hendry (2016). An Overview of Forecasting Facing Breaks. Journal of Business Cycle Research, 12, 3-23.

Clements, M.P. and D.F. Hendry (1996). Intercept Correction and Structural Change. Journal of Applied Econometrics, 11, 475-494.

Hanif, M.N. and M.J. Malik (2015). Evaluating Performance of Inflation Forecasting Models of Pakistan. SBP Research Bulletin. 11 (1). 


\section{Appendix A: Intercept Correction Step by Step}

Intercept correction is a way to adjust for intercept changes in an estimated model of a set of time series variables. For intercept correction purpose we use some data points (say half, $\mathrm{T}=\mathrm{N} / 2$ ) of the available observations to estimate the underlying relationship ${ }^{3}$ without adding intercept and get one period ahead forecast. Since we also have actual data for second half of the data set, we obtain the corresponding forecast error. Then we add a fraction, w (which can be any number greater than zero but usually less than or equal to 1), of this forecast error in the model. One can pick this fraction arbitrarily, say $\mathrm{w}=0.5$ or can select it optimally (one with minimum root mean square error from amongst those with $\mathrm{w}=0.01,0.02, \ldots, \mathrm{w}=1.00)$. We use this model to forecast for the next period. We repeat this process up to the last observation. We in the last, forecast for $\mathrm{N}+1$ period similarly, which actually incorporates the changes in intercept during the previous periods. Here we explain how.

Let us assume that the true data generating process for first half of the sample is

$\mathrm{y}_{\mathrm{t}}=\beta_{0}+\beta_{1} \mathrm{y}_{\mathrm{t}-1}+\beta_{2} \mathrm{x}_{\mathrm{t}-1}+\epsilon_{\mathrm{t}}$

Where $\in \sim \mathrm{N}(0, \Sigma)$

Now we use first $\mathrm{T}$ (=half of the total number of) observations for the estimation of Model (2.1). One step ahead forecast for the $\mathrm{y}$ is;

$\hat{y}_{\mathrm{T}}=\hat{\beta}_{0}+\hat{\beta}_{1} \mathrm{y}_{\mathrm{T}-1}+\hat{\beta}_{2} \mathrm{x}_{\mathrm{T}-1}+\hat{\epsilon}_{\mathrm{t}}$

$\hat{\mathrm{y}}_{\mathrm{T}+1}^{\mathrm{F}}=\hat{\beta}_{0}+\hat{\beta}_{1} \mathrm{y}_{\mathrm{T}}+\widehat{\beta}_{2} \mathrm{x}_{\mathrm{T}}+\hat{\epsilon}_{\mathrm{T}+1}$

Let us assume that the change in intercept $\beta_{0}$ in (2.1) occurs at time $T+1$ and $\beta_{0}$ changes to $\beta_{0}{ }^{*}$ while other parameters remain unchanged. Now $\mathrm{y}_{\mathrm{T}+1}$ is

$\mathrm{y}_{\mathrm{T}+1}=\beta_{0}{ }^{*}+\beta_{1} \mathrm{y}_{\mathrm{T}}+\beta_{2} \mathrm{x}_{\mathrm{T}}+\epsilon_{\mathrm{T}+1}$

In the presence of change in $\beta_{0}$ forecast error would be;

$$
\begin{gathered}
\epsilon^{\mathrm{F}}{ }_{\mathrm{T}+1}=\left\{\beta_{0}{ }^{*}+\beta_{1} \mathrm{y}_{\mathrm{T}}+\beta_{2} \mathrm{x}_{\mathrm{T}}+\epsilon_{\mathrm{T}+1}\right\}-\left\{\widehat{\beta}_{0}+\widehat{\beta}_{1} \mathrm{y}_{\mathrm{T}}+\widehat{\beta}_{2} \mathrm{x}_{\mathrm{T}}+\hat{\epsilon}_{\mathrm{T}+1}\right\} \\
\epsilon_{\mathrm{T}+1}^{\mathrm{F}}=\left\{\beta_{0}{ }^{*}-\widehat{\beta}_{0}\right\}+\left\{\beta_{1}-\widehat{\beta}_{1}\right\} \mathrm{y}_{\mathrm{T}}+\left\{\beta_{2}-\widehat{\beta}_{2}\right\} \mathrm{x}_{\mathrm{T}}+\left\{\epsilon_{\mathrm{T}+1}-\hat{\epsilon}_{\mathrm{T}+1}\right\}
\end{gathered}
$$

In equation (A4) there are four components of $\epsilon^{\mathrm{F}}{ }_{\mathrm{T}+1}$ particularly $\left\{\beta_{0}{ }^{*}-\widehat{\beta_{0}}\right\}$ could be due to [wrong] assumption that $\beta_{0}$ is constant over the forecast horizon. It results in forecast failure ${ }^{4}$. Other three components are just due to the estimation of true parameters.

In the following we illustrate the implementation of intercept correction procedure and the way it helps reduce the forecast error in (A.4).

We use first half observations for the estimation of model (A.1) but without intercept. One step ahead forecast for the $\mathrm{y}$ is;

$\hat{\mathrm{y}}_{\mathrm{T}}=\widehat{\beta}_{1} \mathrm{y}_{\mathrm{T}-1}+\widehat{\beta}_{2} \mathrm{x}_{\mathrm{T}-1}+\hat{\epsilon}_{\mathrm{t}}$

$\hat{\mathrm{y}}_{\mathrm{T}+1}^{\mathrm{F}}=\widehat{\beta}_{1} \mathrm{y}_{\mathrm{T}}+\widehat{\beta}_{2} \mathrm{x}_{\mathrm{T}}+\hat{\epsilon}_{\mathrm{T}+1}$

Assuming that the change in intercept in (A.1), $\beta_{0}$ occurred at $\mathrm{T}+1$ and it became $\beta_{0}{ }^{*}$ all other parameters remain unaffected. Now $\mathrm{y}_{\mathrm{T}+1}$ would be;

$\mathrm{y}_{\mathrm{T}+1}=\beta_{0}^{*}+\beta_{1} \mathrm{y}_{\mathrm{T}}+\beta_{2} \mathrm{x}_{\mathrm{T}}+\epsilon_{\mathrm{T}+1}$

\footnotetext{
${ }^{3}$ For simplicity, we consider only two variables and 1 lag here.

${ }^{4}$ In contrast to forecast failure due to shift in long run mean of the explained variable, change in any (or all) of the other parameters (causes no systematic bias but) increases forecast variance only (Castle et al, 2016).
} 
In the presence of change in $\beta_{0}$ forecast error would be;

$$
\begin{aligned}
\epsilon^{\mathrm{F}}{ }_{\mathrm{T}+1} & =\left\{\beta_{0}{ }^{*}+\beta_{1} \mathrm{y}_{\mathrm{T}}+\beta_{2} \mathrm{x}_{\mathrm{T}}+\epsilon_{\mathrm{T}+1}\right\}-\left\{\widehat{\beta}_{1} \mathrm{y}_{\mathrm{T}}+\widehat{\beta}_{2} \mathrm{x}_{\mathrm{T}}+\hat{\epsilon}_{\mathrm{T}+1}\right\} \\
\epsilon_{\mathrm{T}+1}^{\mathrm{F}} & =\left\{\beta_{0}{ }^{*}\right\}+\left\{\beta_{1}-\widehat{\beta}_{1}\right\} \mathrm{y}_{\mathrm{T}}+\left\{\beta_{2}-\widehat{\beta}_{2}\right\} \mathrm{x}_{\mathrm{T}}+\left\{\epsilon_{\mathrm{T}+1}-\hat{\epsilon}_{\mathrm{T}+1}\right\}
\end{aligned}
$$

In equation (A.7) the component of error " $\left\{\beta_{0}{ }^{*}\right\}$ " could be due to [wrongly] omitting intercept in the estimation. We repeat the above procedure up to all the observations in last half of the data set. Now we have the last N/2 forecast errors and corresponding observations.

In the following we illustrate how to incorporate intercept change by including the forecast error at $\epsilon^{\mathrm{F}}{ }_{\mathrm{N}}$ as a regressor in the model. In doing so we will be ignoring $\beta_{0}$ in the regression on last N/2 observations;

$\hat{y}_{N}=\widehat{\beta}_{1} y_{N-1}+\widehat{\beta}_{2} x_{N-1}+\widehat{w}_{0} \epsilon^{F}{ }_{N-1}+\hat{\epsilon}_{N}$

One step ahead forecast would be,

$\mathrm{y}^{\mathrm{F}}{ }_{\mathrm{N}+1}=\widehat{\beta}_{1} \mathrm{y}_{\mathrm{N}}+\widehat{\beta}_{2} \mathrm{x}_{\mathrm{N}}+\widehat{\mathrm{w}}_{0} \epsilon^{\mathrm{F}}{ }_{\mathrm{N}}+\hat{\epsilon}_{\mathrm{N}+1}$

Substituting $\epsilon^{\mathrm{F}}{ }_{\mathrm{N}}$ in (A9) we have;

$\mathrm{y}^{\mathrm{F}}{ }_{\mathrm{N}+1}=\widehat{\beta}_{1} \mathrm{y}_{\mathrm{N}}+\widehat{\beta}_{2} \mathrm{x}_{\mathrm{N}}+\widehat{\mathrm{w}}_{0}\left[\left\{\beta_{0}{ }^{*}\right\}+\left\{\beta_{1}-\widehat{\beta}_{1}\right\} \mathrm{y}_{\mathrm{N}-1}+\left\{\beta_{2}-\widehat{\beta}_{2}\right\} \mathrm{x}_{\mathrm{N}-1}+\left\{\epsilon_{\mathrm{N}}-\hat{\epsilon}_{\mathrm{N}}\right\}\right]+\hat{\epsilon}_{\mathrm{N}+1}$

$\mathrm{y}^{\mathrm{F}}{ }_{\mathrm{N}+1}=\left(\widehat{\mathrm{w}}_{0}\left(\beta_{0}{ }^{*}\right)\right)+\widehat{\beta}_{1} \mathrm{y}_{\mathrm{N}}+\widehat{\mathrm{w}}_{0}\left(\beta_{1}-\widehat{\beta}_{1}\right) \mathrm{y}_{\mathrm{N}-1}+\widehat{\beta}_{2} \mathrm{x}_{\mathrm{N}}+\widehat{\mathrm{w}}_{0}\left\{\beta_{2}-\widehat{\beta}_{2}\right\} \mathrm{x}_{\mathrm{N}-1}+\widehat{\mathrm{w}}_{0}\left\{\left(\epsilon_{\mathrm{N}}-\right.\right.$ $\left.\hat{\epsilon}_{\mathrm{N}}\right)+\hat{\epsilon}_{\mathrm{N}+1}$

Above equation shows that intercept change at $\mathrm{T}+1$ is incorporated in forecasting $\mathrm{y}_{\mathrm{N}+1}$. Since the above procedure is recursive so we can incorporate the changes occurring at different time periods in our forecast for the variable of interest.

\section{Appendix B}

Figure B.1 Standard Deviation of Inflation and Optimal w0 1992-2016

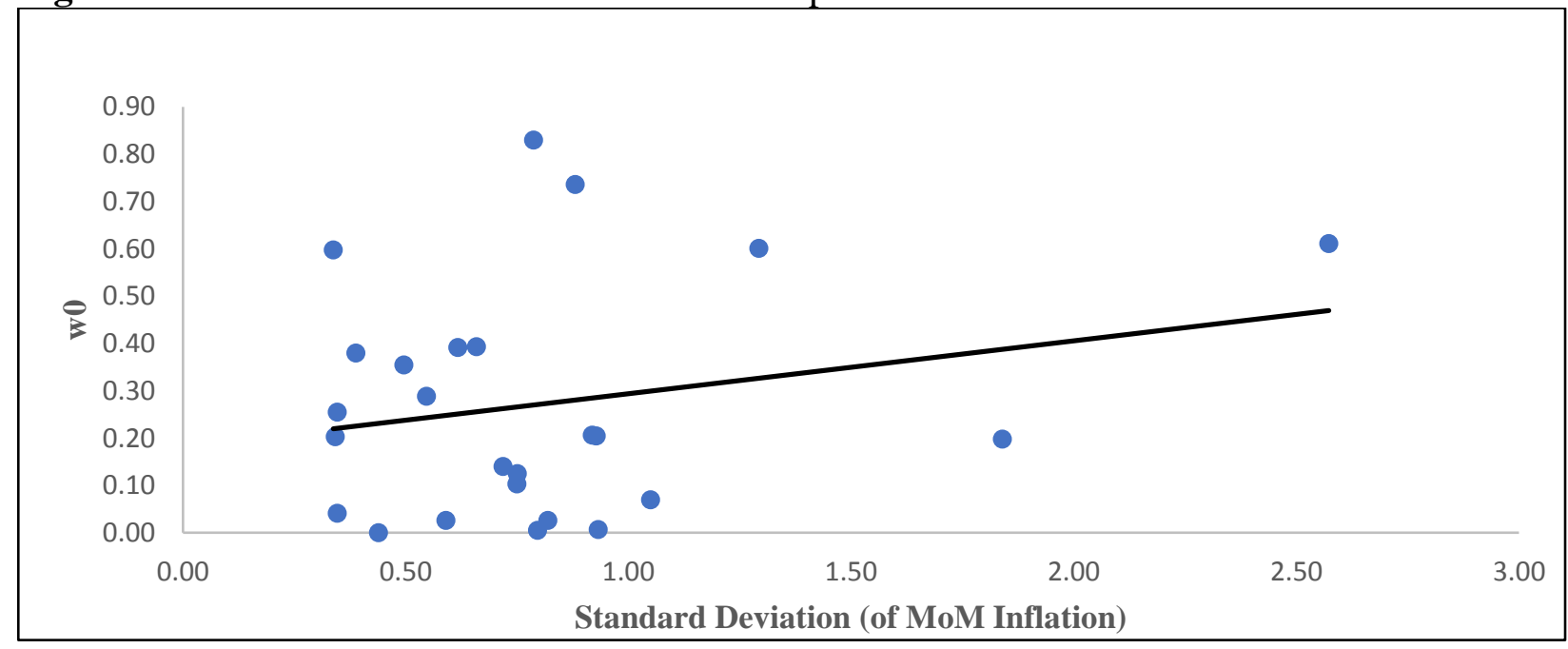


Figure B.2 RMSEs Relative to Random Walk With Drift Against Different Values of w

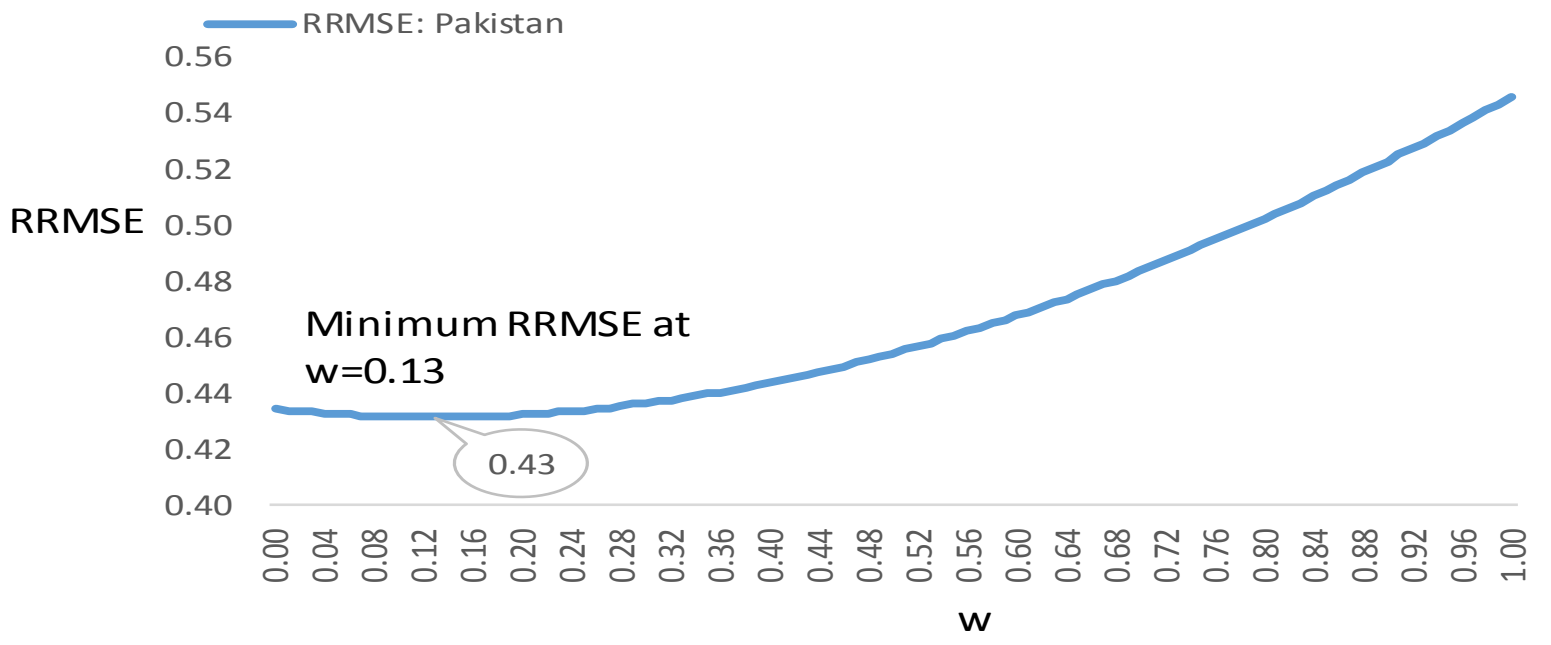


Figure B.3 RMSEs Relative to Random Walk Model with Drift Against different values of $w$.

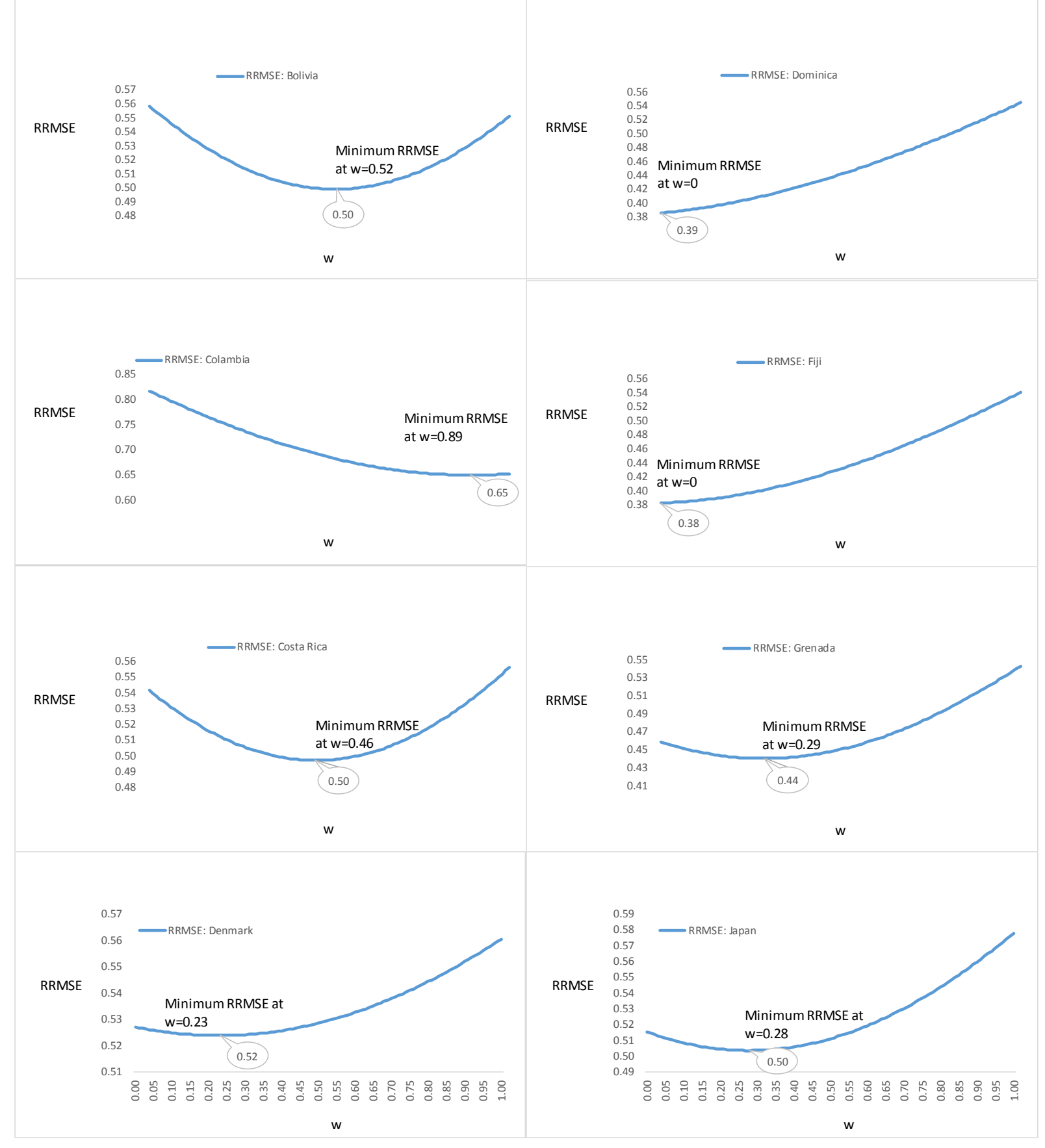


Figure B.4 RMSEs Relative to Random Walk Model with Drift Against different values of $w$.

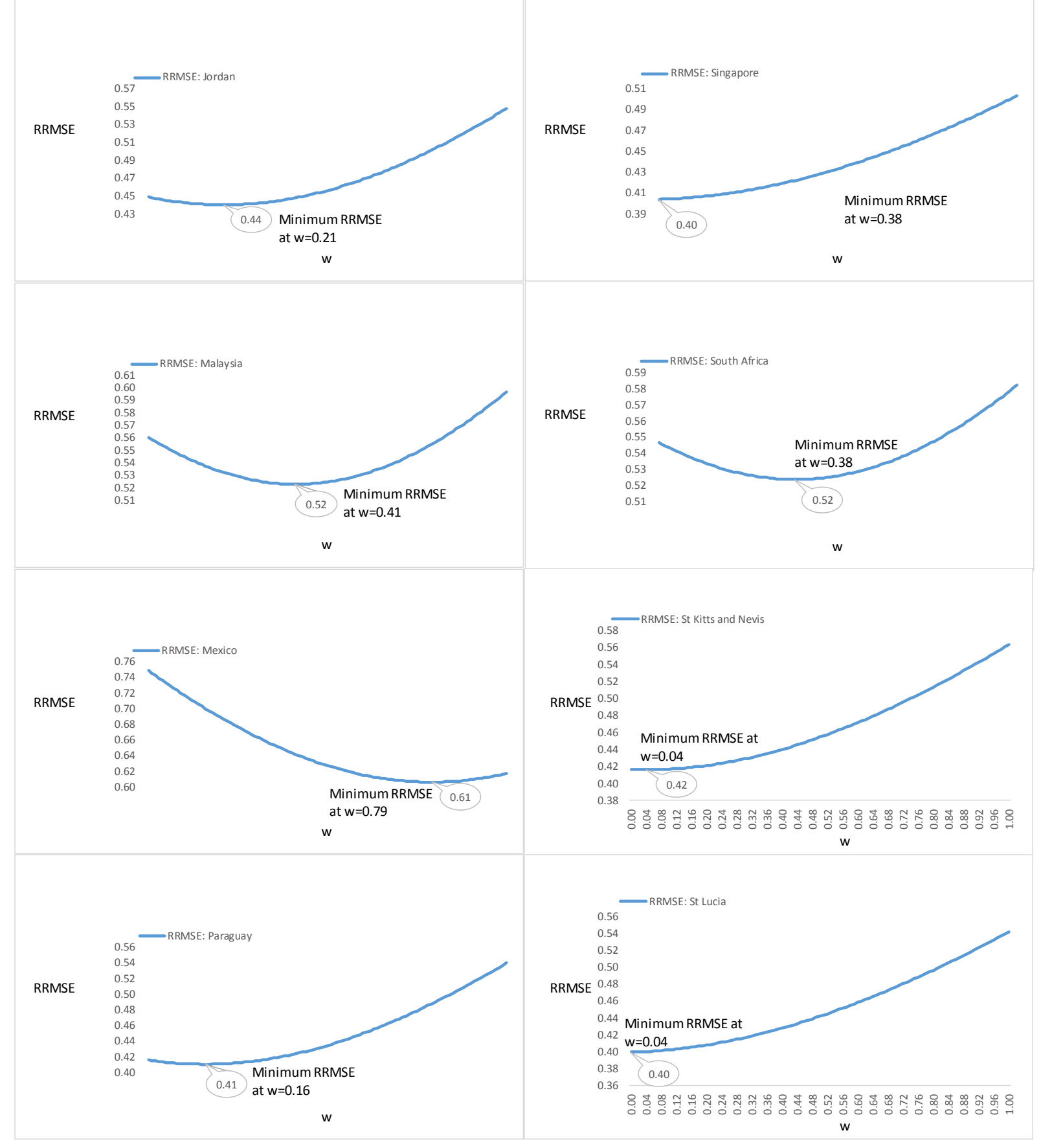


Figure B.5 RMSEs Relative to Random Walk Model with Drift Against different values of $w$.

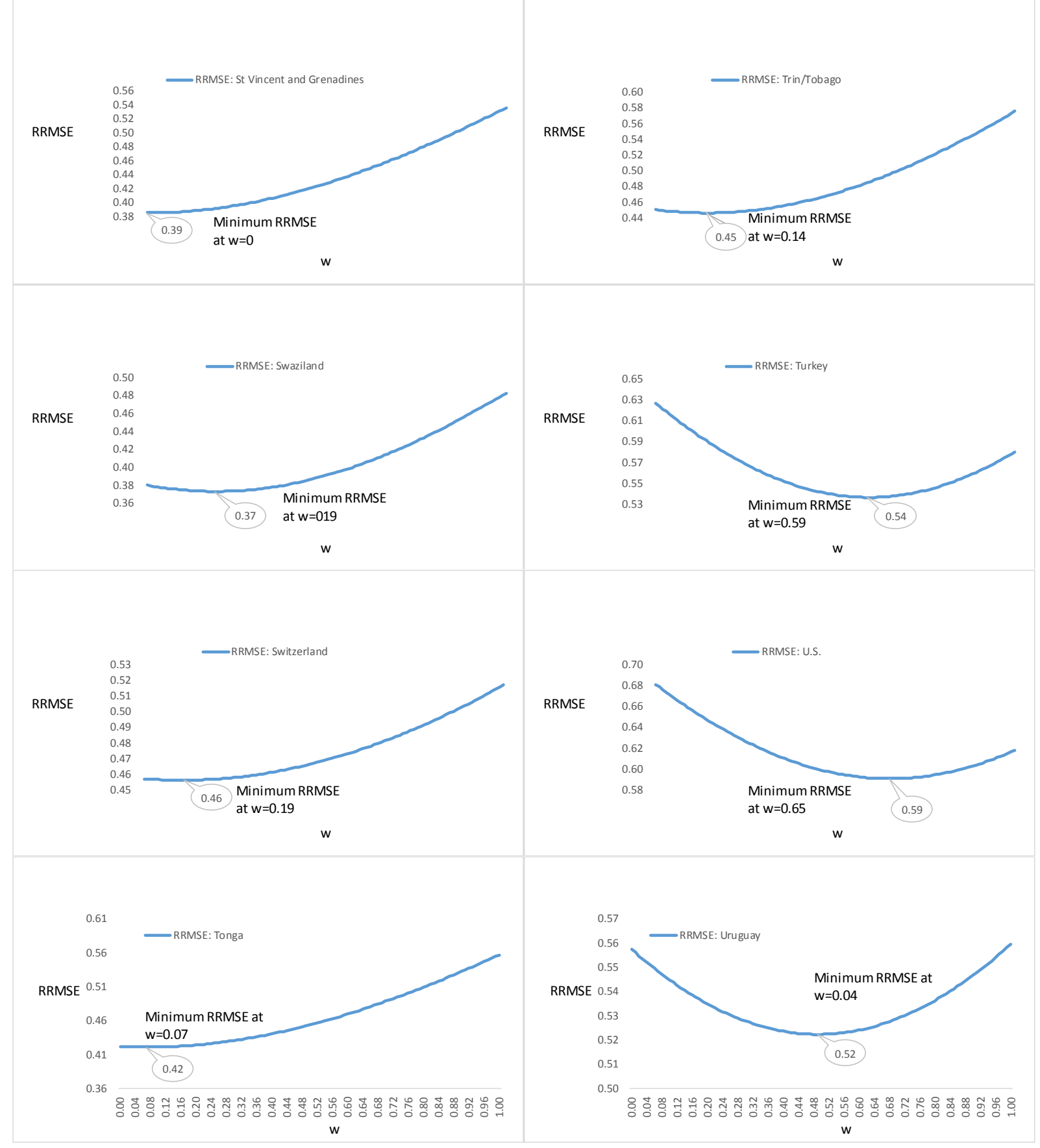


Figure B.6 Country-wise OIC for Different Samples

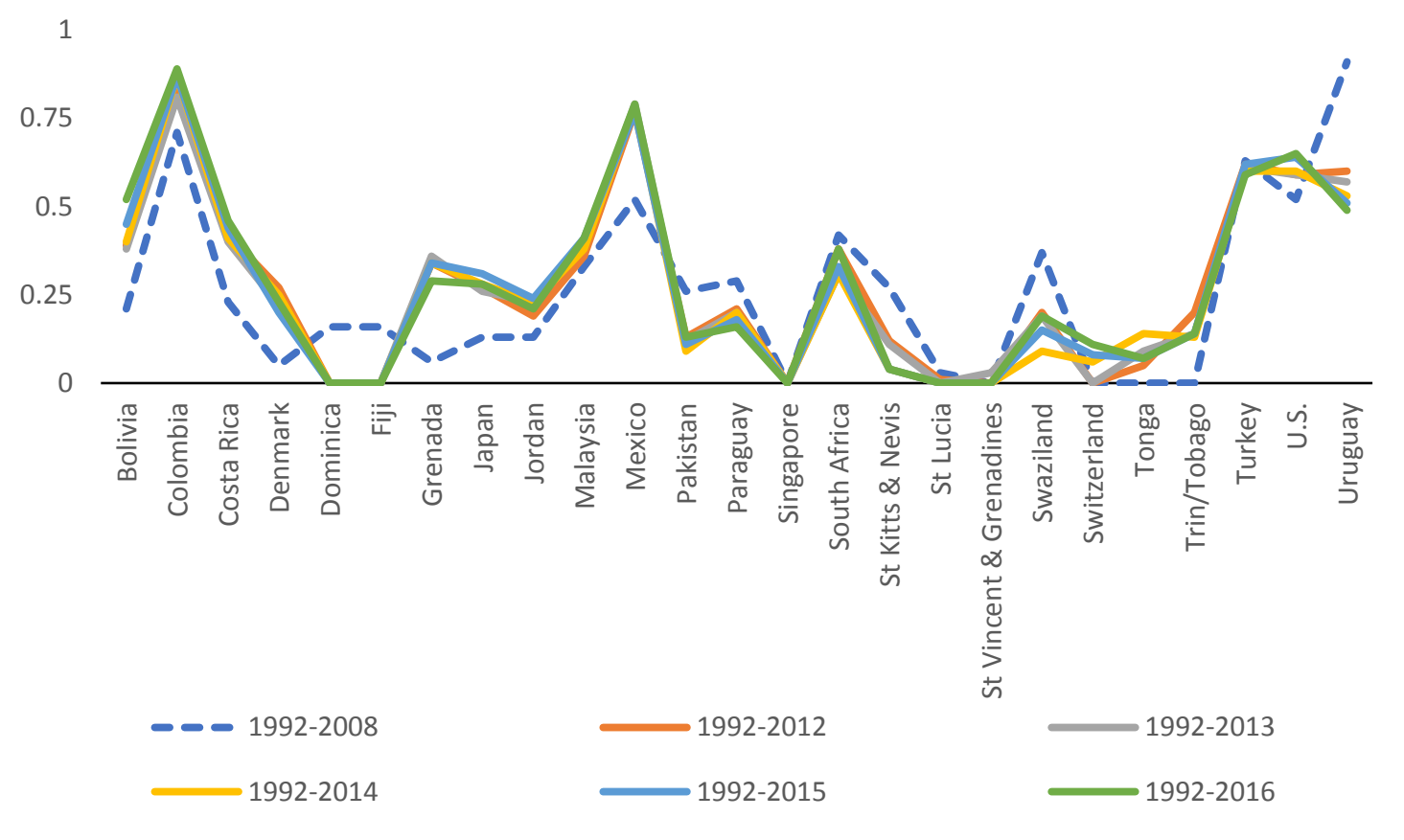

